\title{
Metal artifact reduction on MRI images
}

\author{
Nikolay V. Gapon ${ }^{1,}$, Evgenii A. Semenishchev ${ }^{1}$, Oxana S. Balabaeva ${ }^{1}$, Arina A. Skorikova ${ }^{2}$, \\ Olga A. Tokareva ${ }^{1}$ and Alexey M. Nadykto ${ }^{3}$ \\ ${ }^{1}$ Don State Technical University, 344000 Rostov-on-Don, Russia \\ ${ }^{2}$ Volgograd State Medical University, 400131 Volgograd, Russia \\ ${ }^{3}$ Moscow State University of Technology "STANKIN"(MSTU), 127055 Moscow, Russia
}

\begin{abstract}
This article examines the method of image reconstruction, which aims to restore the exposed areas on MRI images. The algorithm is based on a geometric model for patch synthesis. The lost pixels are recovered by copying pixel values from the source using a similarity criterion. We used a trained neural network to choose the "best similar" patch. Experimental results show that the proposed method outperforms widely used state-of-the-art methods.
\end{abstract}

\section{The statement of the problem}

In modern society, we are confronted with digital images in a variety of situations: from everyday life, where analog cameras have long been replaced by digital ones. Their professional use is medicine, the sciences of earth, art, and security. Examples of medical instruments for imaging are MRI (magnetic resonance imaging), PET (positron emission tomography), CT (computed tomography) for imaging the brain, internal organs, the human skeleton. These visualization tools usually create noisy or image with artifacts. Artifacts are illuminated areas resulting from metal implants in the image. Also, there are many other small items, such as surgical clips, dental fillers, mounting screws, or surgical pins, which can also show as artifacts on MRI scans. When the X-rays reach the metal in the body, they weaken considerably, with the result that fewer photons reach the detector.
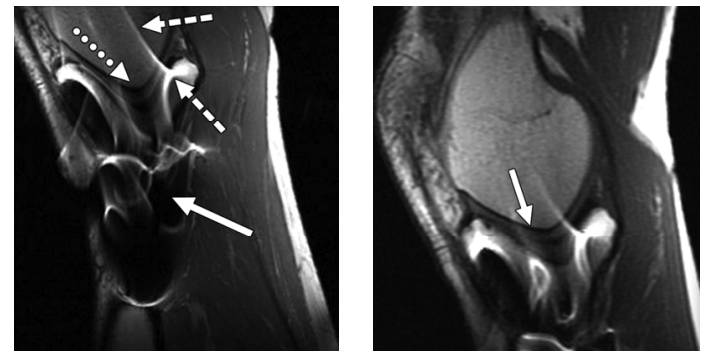

Fig. 1. Examples of artifacts due to the presence of stainless-steel screws.

\footnotetext{
*Corresponding author: gapon.n.v@gmail.com
} 
Nonlinearity caused by a low number of photons causes damage, seriously deteriorating the image quality near metal surfaces and causing the appearance of illuminated areas. Therefore, before they can be evaluated by doctors, they must be processed. This article examines the method of image reconstruction, which aims to restore the exposed areas on MRI images.

\section{Proposed method}

\subsection{Method reconstruction}

At the first step, for each pixel of the boundary $\delta S_{i, j}$ using the inversion method the shape of the domain for similarity search is adaptively determined by combining two adjacent homogeneous sub regions in the direction of the maximum gradient $[2,5,6]$.

The second step calculates the priority value $P(\delta S)$ for each pixel value of the boundary, which consists of two factors $[3,5]$ :

$$
\begin{gathered}
P(\delta S)=C(\delta S) \cdot D(\delta S) \\
C(\delta S)=\frac{\sum_{l \in \Psi_{\delta S}} C(l)}{\left|\Psi_{\delta S}\right|}, D(\delta S)=\frac{\left|\nabla I_{\delta S}^{\perp} \cdot n_{\delta S}\right|}{\alpha}
\end{gathered}
$$

At the third step, we determine blocks $\psi_{q^{(h)}}, h=\overline{1, R}$ in the area of available pixels $S$ for which the Euclidean metric is minimal $[4,6]$ :

$$
\sqrt{\sum\left(\Psi_{p}-\Psi_{q}\right)^{2}} \longrightarrow \min
$$

The pixel values in the region $\eta$ adjacent to the pixel with the highest priority $p$ are reconstructed by averaging the corresponding pixels from chosen areas $\psi_{q^{(h)}}$ in the area of available pixels $S$ using a neural network, in particular, a multi-layer perceptron [6].

The confidence coefficient $C$ for restored pixels is assigned to the current value $C(p)$.

After that, the procedure of priority correction and search of similar areas with subsequent replacement is repeated.

\subsection{Neural network}

In this work, a neural network of direct signal propagation, which was trained using the algorithm for backpropagation of the error was developed. The activation function which is used in this network is the sigmoidal nonlinear function (sigmoidal nonlinearity), namely the hyperbolic tangent function (4)[6].

$$
f(x)=\tanh \left(\frac{a x}{2}\right)=\frac{1-e^{-a x}}{1+e^{-a x}}
$$

$a$ - is the slope parameter of the sigmoidal activation function.

At the training stage of the neural network, pre-prepared data was fed to the input: a block with random coordinates 9x9 in size was allocated to the image, then the central pixel 
was removed, and five most similar blocks were found on the whole image comparing them by MSE.

Then the procedure was repeated on 35 images, and 100,000 blocks were obtained, they were used as a training sequence for this network. This network contains three layers: the first layer contains 20 neurons, the second layer also contains 20 neurons, the third layer contains ten neurons (fig.2).

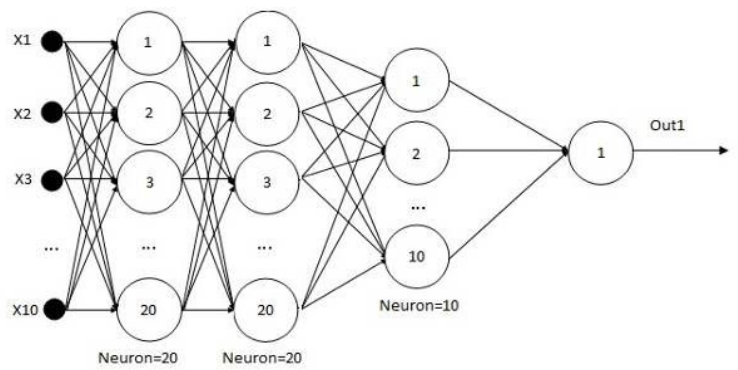

Fig. 2. The architecture of the neural network.

The network created ten inputs, 5 of them were fed only the central pixels in the blocks found, and the other five inputs fed the MSE of these blocks. As an output, there were the central pixels of the original blocks.

\section{The obtained results}

Figure 3 and Figure 4 show examples of image restoration (a) of the original image, b) an image with a mask of distorted pixels, c) an image reconstructed by the proposed method,

d) zoomed image.

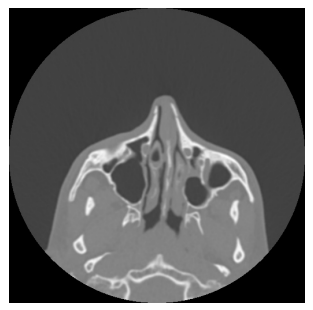

a

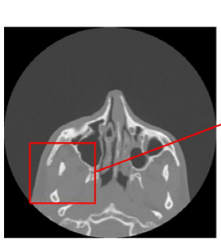

c

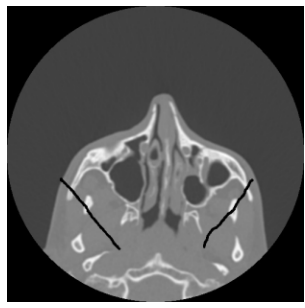

b

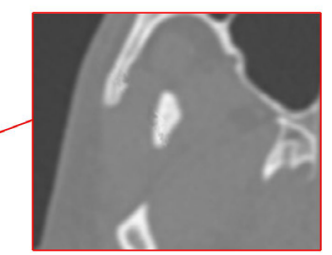

d

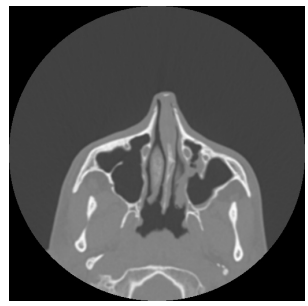

a

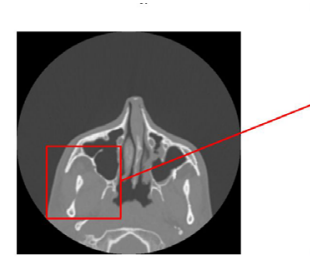

c

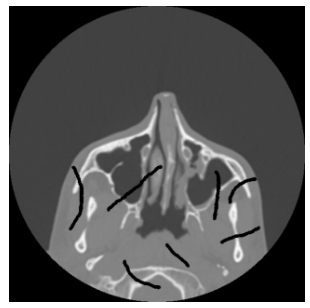

b

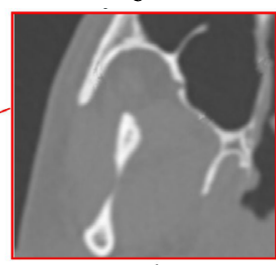

d

Fig.3. Examples of processing: a) original image, b) image with missing area, c) image reconstructed by the proposed method, d) zoomed image.

Fig.4. Examples of processing: a) original image, b) image with missing area, c) image reconstructed by the proposed method, d) zoomed image.

The peculiarity of these test images is that the area of missing pixels located at the intersection of several boundaries. The proposed method of reconstruction of pixel values allows you to "correctly" restore them. 
Figure 5 shows an example of image restoration (a) of the original image, b) an image with a mask of distorted pixels, c) an image reconstructed by the proposed method, d) zoomed image. Also, it should be noted that the proposed method does not blur the texture and structure when restoring large areas with lost pixels.

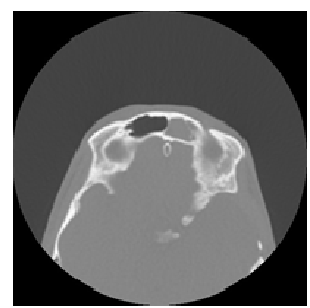

a

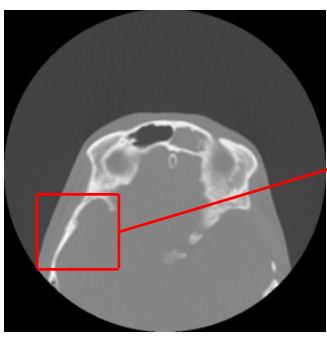

C

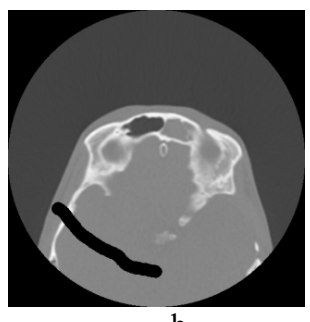

b

Fig. 5. Examples of processing: a) original image, b) image with missing area, c) image reconstructed by the proposed method, d) zoomed image.

The processing error values for the proposed method, EBM [1], Navier Stokes and Telea methods are presented in Table 1. It should be noted that the quantitative values of the errors are calculated in the reconstructed areas. When using the developed image reconstruction method, the error values are on average 10-15\% less than when processed by EBM, Navier Stokes, and Telea.

Table 1. Processing error values.

\begin{tabular}{|l|l|l|l|l|l|l|l|l|l|l|l|l|}
\hline Image & \multicolumn{4}{|c|}{1} & \multicolumn{4}{c|}{$\mathbf{2}$} & \multicolumn{4}{c|}{$\mathbf{3}$} \\
\hline Method & PP & EBM & NS & Telea & PP & EBM & NS & Telea & PP & EBM & NS & Telea \\
\hline PSNR & 30.7 & 28.47 & 26.01 & 25.42 & 25.22 & 24.11 & 25.82 & 23.52 & 36.3 & 31.91 & 28.24 & 26.56 \\
\hline RMSE & 7.41 & 9.61 & 12.75 & 13.65 & 15.18 & 15.34 & 16.36 & 14.92 & 3.9 & 6.46 & 9.87 & 11.97 \\
\hline MAE & 3.94 & 4.92 & 5.94 & 7.31 & 6.09 & 6.9 & 6.08 & 5.77 & 1.8 & 2.49 & 3.6 & 4.48 \\
\hline SNR & 26.1 & 23.83 & 21.37 & 20.78 & 18.58 & 18.27 & 22.18 & 21.88 & 31.18 & 26.79 & 23.11 & 21.44 \\
\hline MAX & 43 & 57 & 79 & 81 & 58 & 117 & 60 & 62 & 35 & 90 & 76 & 85 \\
\hline
\end{tabular}

Figures 6-8 show examples of MRI image recovery. a) original image from artifacts a) image reconstructed by the proposed method.

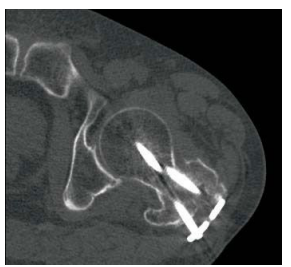

$\mathrm{a}$

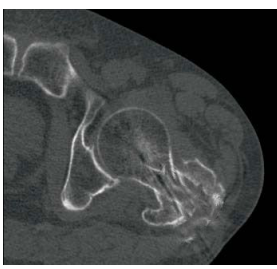

b

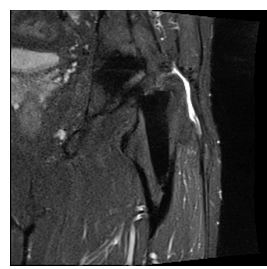

a

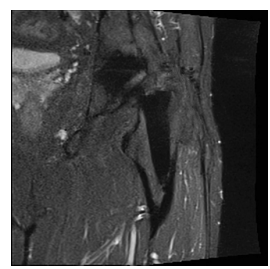

b

Fig. 6. Examples of processing: a) original image

Fig. 7. Examples of processing: a) original image 
from artifacts b) image reconstructed by the proposed method.

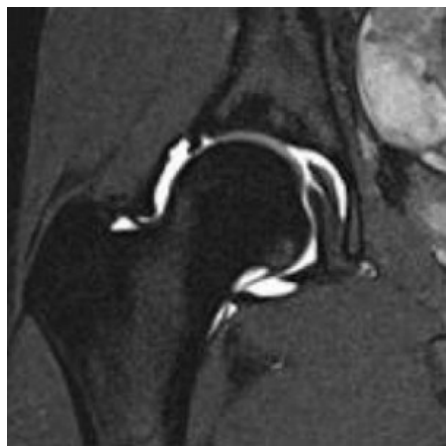

a from artifacts b) image reconstructed by the proposed method.

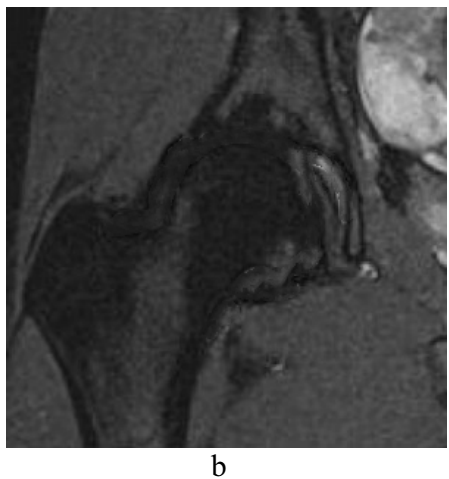

Fig. 8. Examples of processing: a) original image from artifacts b) image reconstructed by the proposed method.

\section{Conclusion}

The results of the recovery of MRI images using the proposed algorithm are presented. The analysis of the image reconstruction method was carried out, which showed that the proposed method makes it possible to efficiently restore artifacts of MRI images. Analysis of the processing results shows that the proposed method allows you to correctly restore the details and background in the image.

We at this moment acknowledge the support of the Ministry of Science and Education of the Russian Federation, in accordance with the decree of the Government of April 9, 2010, №218, project number 03.G25.31.0234.

\section{References}

1. A. Criminisi, P. Perez, K. Toyama, 2003 IEEE Computer Society Conference on Computer Vision and Pattern Recognition, Proceedings (CVPR), 721 (2003)

2. V.V. Voronin, V.I. Marchuk, N.V. Gapon, R.A. Sizyakin, A.I. Sherstobitov, K.O. Egiazarian, Proc. SPIE 9019, Image Processing: Algorithms and Systems XII, 9019, 901907 (2014) doi: 10.1117/12.2038556

3. V.V. Voronin, V.I. Marchuk, N.V. Gapon, A. V. Zhuravlev, S. Maslennikov, S. Stradanchenko, Proc. SPIE 9497, Mobile Multimedia/Image Processing, Security, and Applications, 9497, 94970Y (2015) doi: 10.1117/12.2076509

4. V. V. Voronin, V. I. Marchuk, V. A. Franz, N. V. Gapon, Achievements of Modern Radioelectronics, 6, 31 (2012)

5. V. V. Voronin, V. I. Marchuk, N. V. Gapon, R. A. Sizyakin, Achievements of Modern Radioelectronics, 9, 16 (2011)

6. N. Gapon, M. Pismenskova, O. Tokareva, M. Ponamorenko, MATEC Web Conf, 132, (05015) 